\title{
JMCT Monte Carlo Simulation Analysis of BEAVRS and SG-III Shielding
}

\author{
Deng $\mathrm{Li}^{1,2^{*}}$, Li Gang ${ }^{1,2}$, Zhang Baoyin ${ }^{1,2}$, Shangguan Danhua ${ }^{1,2}$, Ma Yan $^{1,2}$, Hu Zehua $^{1,2}$, Fu Yuanguang ${ }^{2}$, \\ Li Rui ${ }^{2}$, Shi Dunfu, ${ }^{2}$,Hu Xiaoli ${ }^{2}$, Wang Wei ${ }^{1,2}$ \\ 1 Institute of Applied Physics and Computational Mathematics (IAPCM), Beijing, China \\ 2 CAEP Software Centre for High Performance Numerical Simulation(CAEP-SCNS), Beijing, China
}

\begin{abstract}
JMCT is a general purpose Mont Carlo neutron-photon-electron or coupled neutron/photon/electron transport code with a continuous energy and multigroup. The code has almost all functions of a general Monte Carlo code which include the various variance reduction techniques, the multi-level parallel computation of MPI and OpenMP, the domain decomposition and on-fly Doppler broadening, etc. Especially, JMCT supports the depletion calculation with TTA and CRAM methods. The input uses the CAD modelling and the calculated results use the visual output. The geometry zones, materials, tallies, depletion zones, memories and the period of random number are enough big for suit of various problems. This paper describes the application of the JMCT Monte Carlo code to the simulation of BEAVRS and SG-III shielding model. For BEAVRS model, the JMCT results of HZP status are almost the same with MC21, OpenMC and experiment. Also, we performed the coupled calculation of neutron transport and depletion in full power. The results of ten depletion steps are obtained, where the depletion regions exceed 1.5 million and 120 thousand processors to be used. Due to no coupled with thermal hydraulics, the result is only for reference. Finally, we performed the detail modelling for Chinese SG-III laser facility, where the anomalistic geometry bodies exceed 10 thousands. The flux distribution of the radiation shielding is obtain based on the mesh tally in case of Deuterium-Tritium fusion reaction. The high fidelity of JMCT has been shown.
\end{abstract}

\section{INTRODUCTION}

The high fidelity particle transport system JPTS( $\underline{\mathrm{J}}$ Particle Transport System) has been developed for simulation of reactor full-core and shielding. This package is developed based on the three support framework JASMIN ${ }^{[1]}$, JAUMIN and $\mathrm{JCOGIN}^{[2]}$, where JASMIN is an adaptive structured mesh infrastructure, JAUMIN is an adaptive unstructured mesh infrastructure and JCOGIN is a parallel combinatorial geometry infrastructure. The JPTS package can do large scale parallel computation. Here, we mainly introduce a general purpose 3-D Monte Carlo transport code JMCT( Monte Carlo Transport ${ }^{[3]}$. Two models are chosen as test examples, where model one is the BEAVRS which is permitted by MIT Computational Reactor Physics Group in M\&C2013 conference ${ }^{[4]}$. Another model is from the Chinese SG-III laser facility.

For BEAVRS model, we finish the detail modelling and simulation for the full core in hot zero power(HZP) status. $95 \%$ pins have less $1 \%$ standard deviation. The detailed pin-power density distribution, standard deviation etc. are shown. Then, we make the coupled calculation of neutron transport and depletion in full power status. Due to the memory resume too large, the simulation is done in case of $30 / 398$ axial plane, where the depletion regions exceed 1.5 million. The simulation uses 120 thousand processors in Chinese TianHe-II supercomputer. Since the BEAVRS model involves the coupled with thermal hydraulics. At present, JMCT isn't coupled with thermal dynamic (being done), so the result is only for reference.

For SG-III model, the geometry is very complicated and irregular; the total geometry bodies exceed ten thousand. We order built some special body, such as optical instrument. The neutron and photon flux distributions of all building are given in case of the deuterium-tritium fusion reaction. The result has been used for the theory evidence of shielding design.

\section{INTRODUCE OF JPTS PACKAGE AND JMCT MONTE CARLO CODE}

\section{1 JPTS Package}

JPTS package is developed by IAPCM. It contains the four applied codes JNuDa, JSNT, JMCT, JBURN and a suit of data libratory $\mathrm{NuDa}$. Furthermore, the $\mathrm{CAD}$ pre-processor JLAMT and view post-processor TeraVAP are equipped(see Figure1). Where:

\footnotetext{
Corresponding author: deng li@iapcm.ac.cn

This work was performed under the National Energy Administration of China (2015ZX06002008) and the Technology and Industry for National Defense (C1520110002).
} 


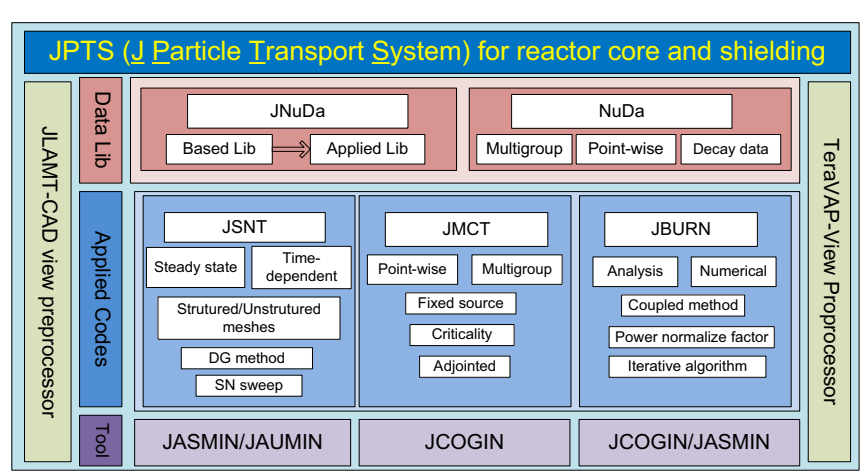

Fig. 1. JPTS package flow

Currently, JSNT $\mathbf{S}_{\mathbf{N}}$ Code Support ${ }^{[5,6]}$

1) Particle type: neutron, photon or coupled of neutron and photon.

2) Mode: forward/adjoint.

3) Problem: fixed-source/criticality.

4) Space: $3 D / 2 D$, Cartesian /cylindrical geometry.

5) Mesh: non-uniform structured (JSNT-S)/unstructured mesh (JSNT-U).

6) Energy: multigroup, anisotropic $\mathrm{P}_{\mathrm{N}}$ scattering $(\mathrm{N}=1,3$, 5).

7) Parallelization: massive parallel computing (spaceangle parallelization).

8) Algorithm: acceleration algorithms (rebalance methods, multigrid methods).

9) Input: visualization modelling and automatic mesh generation, multiple choice of spatial discretion (TWD, DFEM ...).

10) Output: visualization analysis.

11) Support Framework: JASMIN ${ }^{[1]}$.

\section{Currently, JBURN Burnup Code Support}

1) Analysis: Transmutation Trajectory Analysis (TTA).

2) Numerical: Chebyshev Rational Approximation Method (CRAM).

3) Mode: inner coupled with JMCT.

4) Depletion regions: $>1$ millions.

5) Support Framework: JCOGIN ${ }^{[2]}$.

\section{Currently, JNuDa Cross-Section Code Support}

Continuous point-wise/Multigroup/Decay Data, where

1) Point-wise cross-section about 450 nucleus.

2) Multigroup library of $47 / 172$ group for neutron and 20 groups for gamma.

3) Decay data (>1500 elements).

\section{Currently, JLAMT Pre-processor Support ${ }^{[7]}$}

1) Geometry: sphere, cylinder, rectangle, et al., some special geometrical body can be ordered.

2) Repeat structure: especial supporting the same geometry with the different material.

Currently, TeraVAP Post-processor Support

Scale: TB scale data and parallel visualization output.

\subsection{JMCT Monte Carlo Code ${ }^{[3]}$}

JMCT is a general purpose 3-D Monte Carlo transport code of neutron, photon, electron or coupled neutron/photon/ electron with the combinatorial geometry. Currently, JMCT support:

1) Particle type: neutron, photon, electron and coupled neutron/photon/electron.

2) Mode: forward/adjoint/burnup.

3) Problem: fixed-source/criticality.

4) Space: 3D combinatorial geometry.

5) Energy: continuous/multigroup $\left(\mathrm{P}_{5}\right)$.

6) Source: standard source/pin-by-pin source/user defined source.

7) Tally: point/surface/cell/mesh

8) Algorithms: domain decomposition ${ }^{[8]} /$ uniform tally $^{2}$ density $^{[9]} /$ mesh tally and mesh windows. etc.

9) Parallelization: MPI (particle)+OpenMP (domain).

10) Input/Output: CAD modelling and visualization.

11) Temperture: on-fly Doppler broadening ${ }^{[10]}$.

12) Fast critical search of boron concentration: only one step.

13) Tally types: keff, point/surface/volume flux, energy deposition, power and reactivity etc.

14) Support Framework: JCOGIN ${ }^{[2]}$.

\section{TESTS}

\subsection{BEAVRS Model}

\subsubsection{Introduction of Model}

The BEAVRS model was released by MIT Computational Reactor Physics Group in July 7, 2013 (www.crpg.mit.edu). It includes detailed specification of operating 4-loop Westinghouse PWR (3411MW), two cycles of measured data, HZP/full power data, fuel loads by assembly as built, three enriched fuels $(1.6 \%, 2.4 \%$ and $3.1 \%)$. The detailed data is in reference ${ }^{[4]}$. Two cycles of measured data can be used to validate high-fidelity core analysis codes. The basic data is as following:

Fuel assemblies: 193.

Axial planes: 398.

Pins/assembly: $289(17 \times 17$, where 264 fuel pins and 25 guide tubes).

Total tally regions: $22,199,246(193 \times 17 \times 17 \times 1 \times 398)$.

Total regions: $44,398,492(193 \times 17 \times 17 \times 2 \times 398)$.

Requirement: $\leq 1 \%$ standard deviation for $95 \%$ fuel pin-powers.

The part results of $\mathrm{MC} 21^{[11]}$ and OpenMC ${ }^{[12]}$ were presented in PHYSOR2014 ${ }^{[13]}$.

\subsubsection{Simulated Results}

\section{(1) Results in HZP status}

HZP status is simulated in 398 layers in axial direction. Due to the memory exceeding the limit of single core, the space domain is decomposed into 8 parts(figure 2(f)). Eight pin types(figure 2(a)) and nine types of assembles(figure 2(b)). The tally was for all pin fuel regions, the simulation tracks 4 
million neutrons each cycle, it discards 400 cycles of 1000 cycles. Table 1 shows the standard deviation distribution in $95 \%$ confidence level for all of pins. Table 2 shows the keff comparison of JMCT, OpenMC and MC21 in different location of control rods and boron concentrations. Table 3 shows the reactivity worth of control rods in 556K. Figure 3 shows the comparison of pin-power distribution, difference at axial elevation of peak power and the comparisons of the MC21 and JMCT powers in axial. The maximal difference is $3.17 \%$. Figure 4 shows the detectors tallies in meter pipes between JMCT and experiment. The maximal difference is $-14.77 \%$ in B13 assemble and minimum power assemble is $-5.648 \%$ in L15 assemble. Figure 5 shows the axial power shape of the B13(maximal difference) and L15(minimum power assembles) between MC21 and JMCT as compared to experiment.

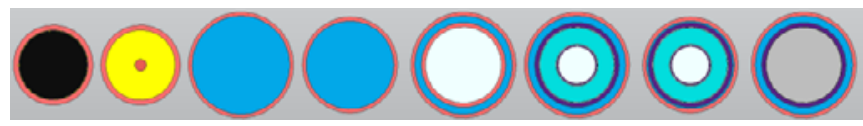

(a) Pins (eight types)

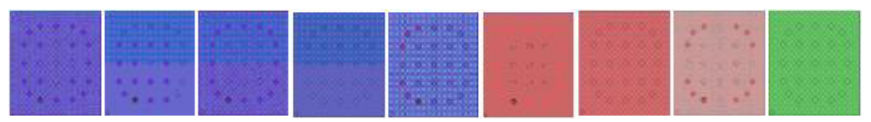

(b)assembles (nine types)

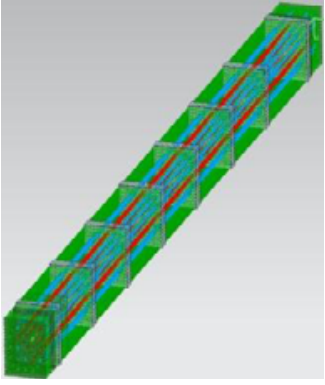

(c)grid

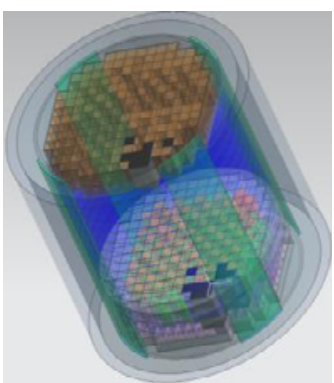

(e)full core

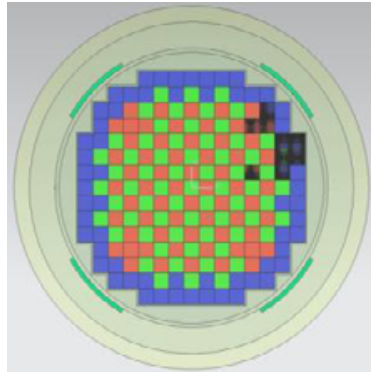

(d)core in radial

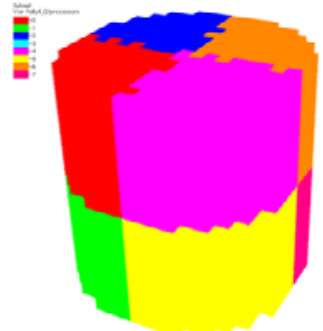

(f) $2 \times 2 \times 2$ domain decomposition
Fig.2. BEAVRS modelling by JLAMT

Table 1. Max and min pin error of flux and energy deposition

\begin{tabular}{|c|c|c|c|c|}
\hline Count & MAX & MIN & $95 \%$ & $99 \%$ \\
\hline Flux & 0.0091 & 0.00118 & $<0.00332$ & $<0.00423$ \\
\hline $\begin{array}{c}\text { Energy } \\
\text { deposition }\end{array}$ & 0.01933 & 0.00254 & $<0.0075$ & $<0.00955$ \\
\hline
\end{tabular}

Table 2. keff comparison in different control rod statuses and boron concentration

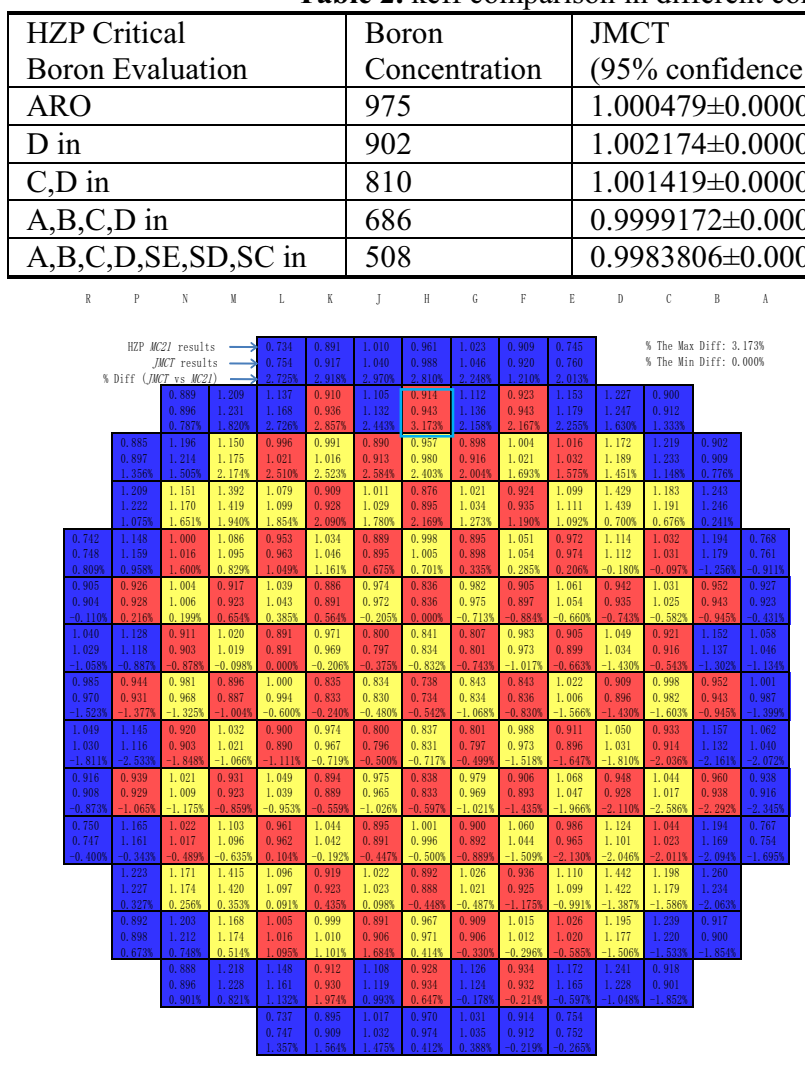

Fig. 3. Comparison of pin power distribution and difference at axial elevation of peak power between MC21 and JMCT

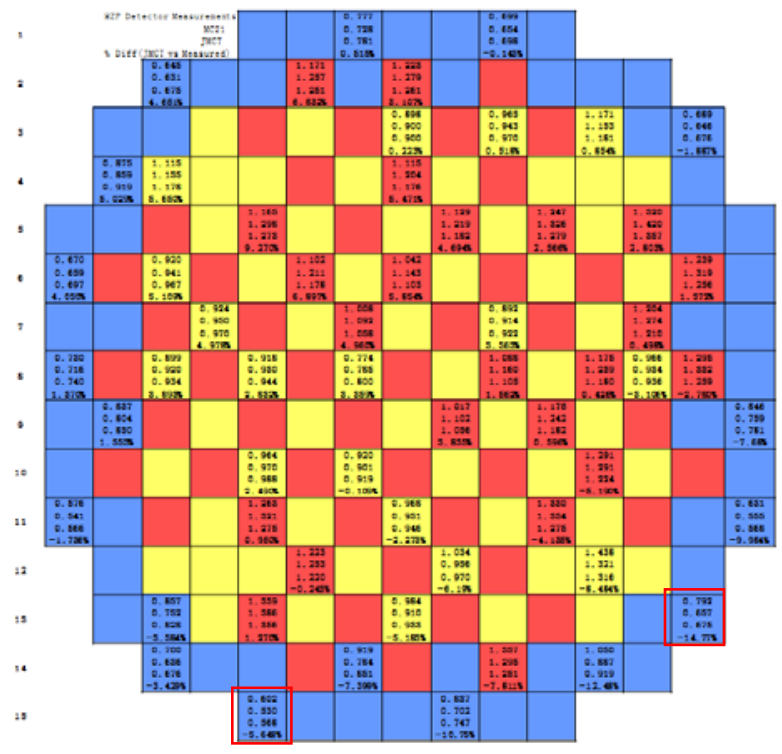

Fig. 4. Comparison of the detectors tallies in meter pipes 
Table 3. Comparison of reactivity worth of control rod in different statuses and boron concentration

\begin{tabular}{|l|l|l|l|l|l|}
\hline HZP Bank worth & Boron & Measure & MC21 & OpenMC & JMCT \\
\hline D & 938.5 & 788 & 773 & $771 \pm 6$ & $770 \pm 6$ \\
\hline C with D in & 856 & 1203 & 1260 & $1234 \pm 7$ & $1258 \pm 6$ \\
\hline B with D,C in & 748 & 1171 & 1172 & $1197 \pm 7$ & $1162 \pm 6$ \\
\hline A with D,C,B in & 748 & 548 & 574 & $556 \pm 6$ & $578 \pm 6$ \\
\hline SE with D,C,B,A in & 597 & 461 & 544 & $501 \pm 6$ & $543 \pm 6$ \\
\hline SD with D,C,B,A,SE in & 597 & 772 & 786 & $844 \pm 6$ & $781 \pm 6$ \\
\hline SC with D,C,B,A,SE,SD in & 597 & 1099 & 1122 & $1049 \pm 6$ & $1107 \pm 6$ \\
\hline
\end{tabular}

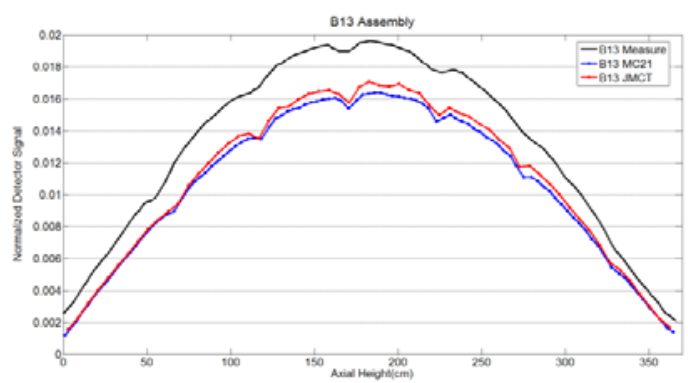

(a)axial power shape in B13 assembly (with maximal difference)

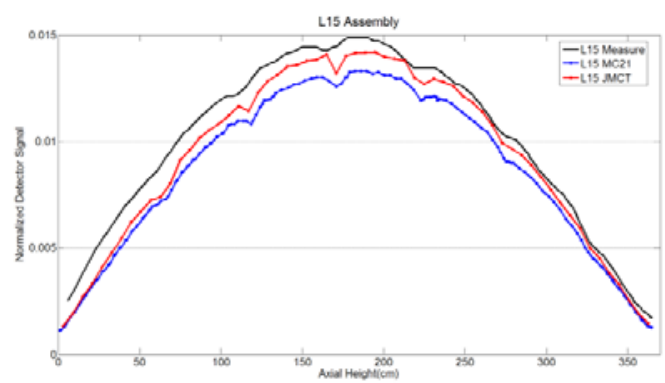

(b)axial power shape in L15 assembly (with minimum power)

Fig. 5. The axial power shape comparison of MC21, JMCT and experiment

\section{(2)Result in Full Power Status}

The coupled neutron transport and depletion is run in $30 / 398$ axial planes, where the depletion region is up to 1528560 $(193 \times 264 \times 30)$. The space domain is also decomposed into 8 parts. It takes about one hour with 120,000 cores on Chinese TianHe-II computer. The result of the tenth steps is obtain. Figure 6 shows the power distribution of some status. Due to the JMCT no coupled with thermal hydraulics, the result is only for reference. For full simulation of BEAVRS model, due to the large memory resume, we predict that the goal need a long time and impossible before 2018.

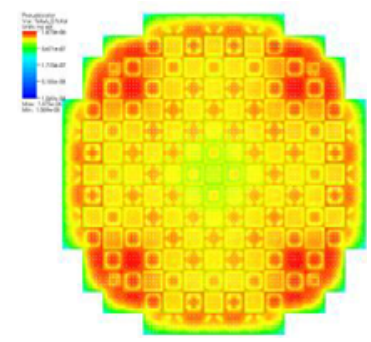

(a)pin power in radial

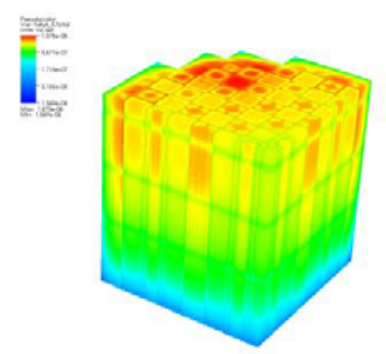

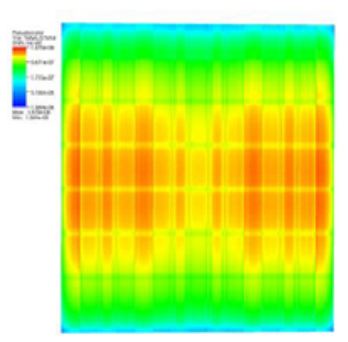

(b)pin power in axial

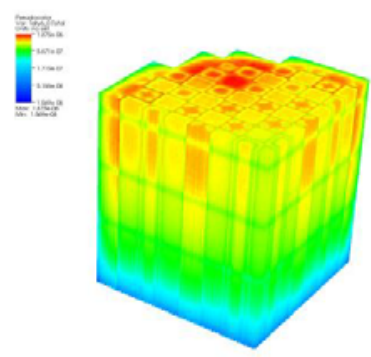

(c)pin power distribution in other status Fig.6. Pin power distributions in full power

\subsection{Chinese SG-III Laser Model}

Chinese SG-III laser device is with 48 laser beams and size in $45 \mathrm{~m} \times 45 \mathrm{~m} \times 53 \mathrm{~m}$. Diameter of target chamber is $6 \mathrm{~m}$. Power is $50 \mathrm{TW}$. It is applied to drive the nuclear fusion reaction by the laser energy. Figure 7 shows the modelling by JMCT pro-processor JLAMT, where figure 7(a)(b) show the locations of tally floors and figure 7(c) shows the building (six floors in ground). The tally is for all floors (seven floors in total). Mesh tally does and it has about 0.63 million meshes. The 0.4 billion neutron histories are simulated by 1024 cores. The $3.1 \mathrm{CPU}$ hours are taken. Where the source is a $14.1 \mathrm{MeV}$ deuterium and tritium(D-T) neutron point source. Figure 8(a)-(b) gives the neutron and photon flux distributions in the base of the fourth floor. Figure 8(c) gives the energy distributions of each floor. Figure $8(d)$ gives a part of flux distribution in building.
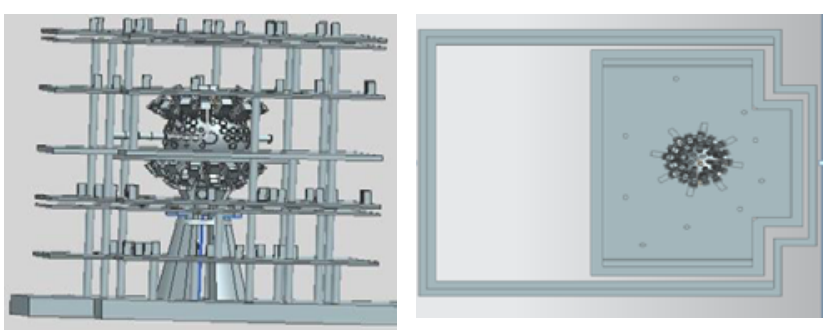

(a)location of the tally floors

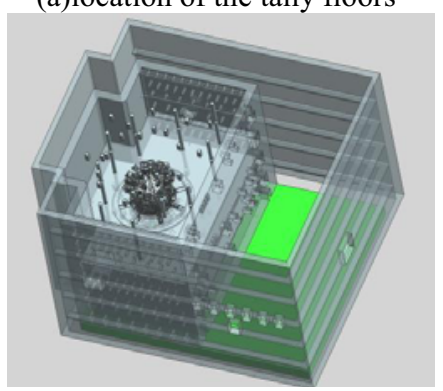

(b)building of SG-III device

Fig.7. Section of SG-III laser devic 


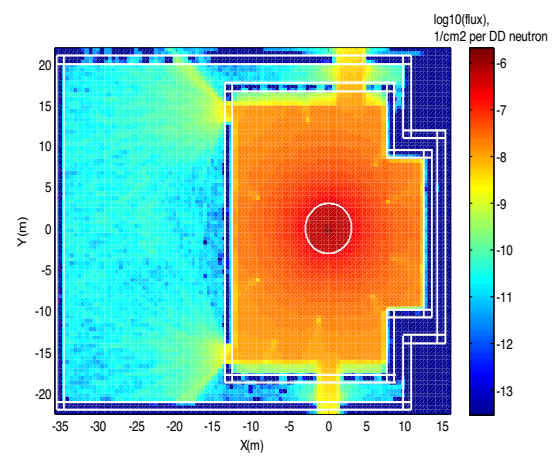

(a)neutron flux in horizontal plane

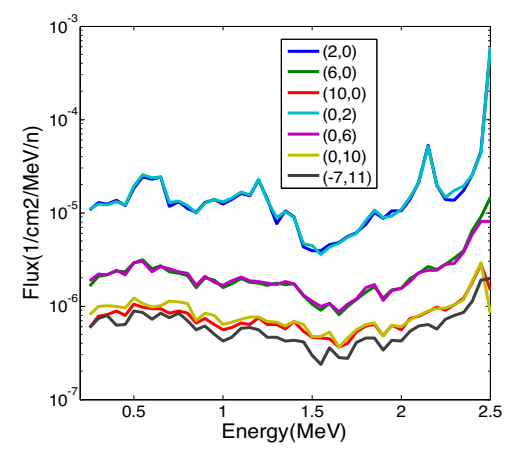

(c)neutron energy spectrum in each floor

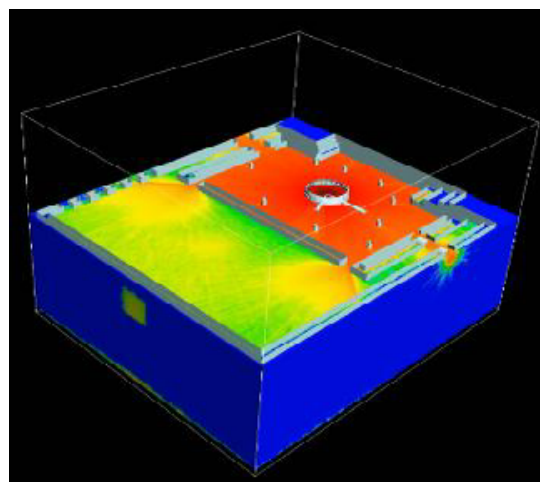

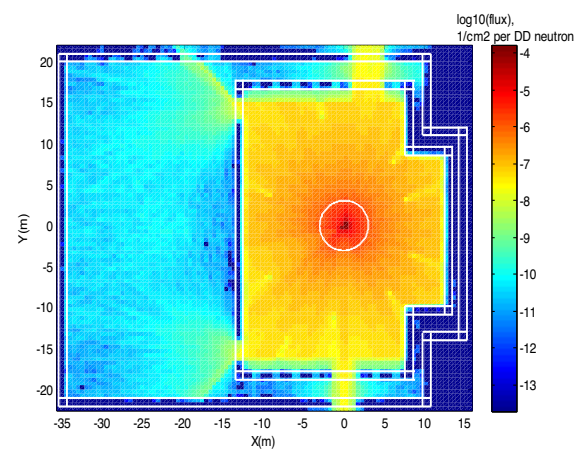

(b)photon flux in horizontal plane

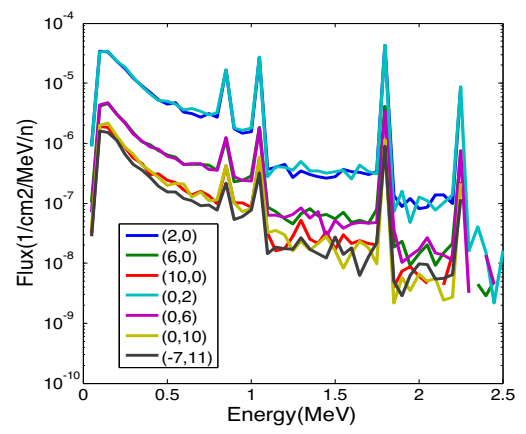

(d)photon energy spectrum in each floor

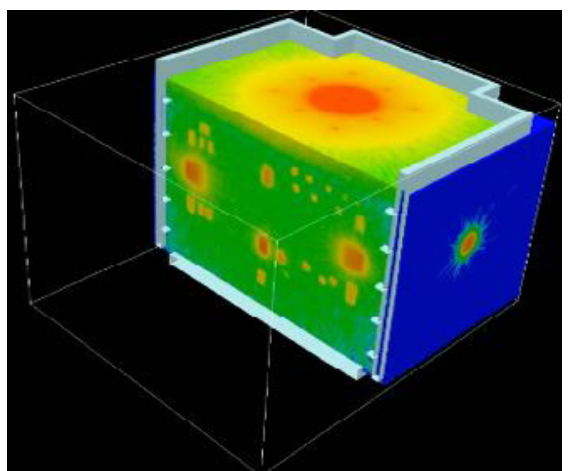

(e)flux distribution of full building (moving)

Fig.8. Flux and energy spectrums in D-T reaction for SG-III model

\section{Conclusion}

JMCT Monte Carlo code is developed and with the capability of the full-core pin-by-pin simulation. It well suits to simulate the large nuclear power reactors and radiation shielding of some large facilities. At present, JMCT still exist no symmetry in power distribution after several burnup steps. We are analysing the reason. On the other hand, the depletion complicating uncertainty quantification and propagation of error will be considered in our next work. Furthermore, it needs to find some new methods to reduce the computational fee. At present, some challenges still exist in simulation of the BEAVRS model. Some new algorithms are being developed.

\section{References}

1. Z. Mo, A. Zhang, X. Cao, et al. "JASMIN: a parallel software infrastructure for scientific computing”. Front. Compute. Sci. China, 2010, 4(4): 480-488.

2. Baoyin Zhang, Gang Li, Li Deng, et al., "JCOGIN: A PARALLEL PROGRAMMING INFRASTRUCTURE FOR MONTE CARLO PARTICLE TRANSPORT," PHYSOR 2014 - The Role of Reactor Physics Toward a Sustainable Future The Westin Miyako, Kyoto, Japan, September 28 October 3, 2014. 
3. Li Deng, Tao Ye, Gang Li et al., "3-D Monte Carlo Neutron-Photon Transport Code JMCT and Its Algorithms", PHYSOR 2014, Kyoto, Japan, September 28 - October 3, 2014.

4. "MIT Benchmark for Evaluation And Validation of Reactor Simulations," RELEASE rev. 1.0.1, MIT Computational Reactor Physics Group, July 7, 2013.

5. Tangpei Cheng, Junxia Wei, Guangchun Zhang, Huayun Shen, Li Deng. Acceleration of Discrete Ordinates Calculations using Parallel Partial Current Rebalance Algorithm and Algebraic Multigrid Solver. The Physics of Reactors conferences (PHYSOR 2016), American Nuclear Society, 2016.

6. Tangpei Cheng, Junxia Wei, Huayun Shen, Bin Zhong, Li Deng. Development of Parallel 3D Discrete Ordinates Transport Program on JASMIN Framework. The 7th International Conference on Modelling and Simulation in Nuclear Science and Engineering, Canadian Nuclear Society, 2015.

7. Yan Ma, YuanGuang Fu, GuiMing Qin, et al, "The large-scale auto modelling tool for Monte Carlo simulations," 7th International Conference on Modelling and Simulation in National Science and Engineering, 2015, October 18-21, Ottawa, Canada
8. G. Li, B. Zhang, L. Deng et al. "Combinatorial Geometry Domain Decomposition Strategies for Monte Carlo Simulations," M\&C 2013, Sun Valley, Idaho, May 5-9, pp.434-443(2013).

9. Danhua ShangGuan, Gang Li, Baoyin Zhang, Li Deng, et al., "Uniform Tally Density-Based Strategy for Efficient Global Tallying in Monte Carlo Criticality Calculation," NUCLEAR SCIENCE AND ENGINEERING, 182(4), 555-562, 2016.

10. Liu Xiong-Guo, Deng Li, Hu Ze-Hua et al., "Study of on-the-fly Doppler broadening in JMCT Program," Acta Phys. Sin., 65(9), 092501-092505, 2016.

11. D. P. Griesheimer, et al., "MC21 v.6.0 - $\mathrm{A}$ Continuous-Energy Monte Carlo Transport Code with Integrated Reactor Feedback Capabilities," Ann. Nucl. Energy, in press, 2015.

12. P.K. Romano, et al., "OpenMC: A state-of-the-art Monte Carlo code for research and development," Ann. Nucl. Energy, in press, 2015.

13. Daniel J. Kelly, Bryan R. Herman, et al., "Analysis of Select BEAVRS PWR Benchmark Cycle 1 Results Using MC21 and OpenMC," PHYSOR 2014, Kyoto, Japan, September 28 October 3, 2014. 